\title{
(6) OPEN ACCESS \\ Does cognitive impairment influence outcomes from cataract surgery? Results from a 1-year follow-up cohort study
}

\author{
Joanna Mary Jefferis, ${ }^{1}$ John-Paul Taylor, ${ }^{2}$ Michael Patrick Clarke $^{3}$
}

\begin{abstract}
${ }^{1}$ Clinical Ageing Research Unit Newcastle University, Newcastle upon Tyne, UK ${ }^{2}$ Institute for Ageing and Health, Newcastle University, Newcastle upon Tyne, UK ${ }^{3}$ Newcastle Eye Centre, Royal Victoria Infirmary, Newcastle upon Tyne, UK
\end{abstract}

\section{Correspondence to} Dr J M Jefferis, Clinical Ageing Research Unit, Campus for Ageing and Vitality, Newcastle University, Newcastle upon Tyne NE4 5PL, UK: joanna.jefferis@ncl.ac.uk

Received 9 June 2014 Revised 4 August 2014 Accepted 25 August 2014 Published Online First 6 October 2014

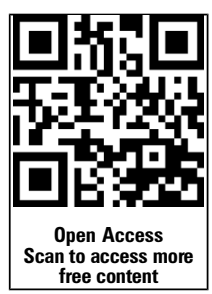

CrossMark

To cite: Jefferis JM, Taylor J-P, Clarke MP. Br J Ophthalmol 2015;99: 412-417.

\section{ABSTRACT}

Background/aims To assess the impact of impaired cognition on visual outcomes 1 year following cataract surgery in a cohort of older people.

Methods Participants aged 75 years or more with bilateral cataract and scheduled for cataract surgery were recruited consecutively. Cognition was assessed using the revised Addenbrooke's cognitive examination (ACE-R). Participants were divided into two groups: normal (ACE-R $\geq 88$ ) and impaired cognition (ACE-R $<88$ ). Visual quality of life (VQOL) and logarithm of minimum angle of resolution visual acuity (VA) were assessed at baseline and 1 year following cataract surgery.

Results of 112 participants, 48 (43\%) had normal cognition and 64 (57\%) had impaired cognition. One year following cataract surgery participants in both groups had significant improvements in VQOL and VA. Visual outcomes at 1 year were significantly better in participants with normal cognition than in those with impaired cognition (95\% Cls for difference $0.4-7.0$ and 0.02-0.1, for VQOL and VA, respectively). Regression analyses correcting for potential confounders showed a relationship between baseline cognition and VA at 1 year $\left(R^{2}=0.30, p=0.001\right)$ and a possible relationship between baseline cognition and VQOL at 1 year $\left(R^{2}=0.41, p=0.01\right.$, this became insignificant after removal of outliers).

Conclusions Patients with impaired cognition benefit from cataract surgery, but not to the same extent as patients with normal cognition.

\section{INTRODUCTION}

Cataract and cognitive impairment are both age-related health problems which rise in prevalence as populations age. Cataract is the leading diagnosis for ambulatory (outpatient) surgery visits in the USA ${ }^{1}$ and cataract surgery is the most common elective surgical procedure carried out in the UK National Health Service. ${ }^{2}$ The prevalence rate of dementia in people aged 75-79 years is $\approx 6 \%$ and double that in the $80-84$ age group. ${ }^{3}$ The prevalence of mild cognitive impairment (MCI) is reported as up to $42 \%$ in older populations. ${ }^{4}$ Therefore, in cataract clinics, where many of the patients attending are in older age groups, one would expect to see large numbers of people with dementia and MCI. For example, a cataract surgeon operating on 20 patients a week would be expected to operate on $\approx 50$ patients with dementia each year. ${ }^{i}$ It is, therefore, important to understand the implications of cognitive impairment on visual acuity (VA) and visual quality of life (VQOL) outcomes for people undergoing cataract surgery.

Older age is known to limit improvements from cataract surgery, even when other ocular comorbidities have been taken into account. ${ }^{5}$ We have previously hypothesised that this may, in part, be due to cerebral ageing and, therefore, postoperative outcomes may be further limited by the additional presence cognitive impairment. ${ }^{7}$ The aim of this study, therefore, was to assess whether cognitive function influenced visual outcomes from cataract surgery in a cohort of older people.

\section{METHODS}

This research adhered to the tenets of the declaration of Helsinki. Ethics committee approval was obtained from the County Durham and Tees Valley research ethics committee.

\section{Participants}

Participants were recruited consecutively from preassessment cataract clinics at a single study centre in the North East of England between March 2011 and August 2012. Participants were eligible for inclusion if they were aged 75 years or more, had bilateral cataract, were scheduled for first eye cataract surgery, had no ocular comorbidity, had no visually significant age-related macular changes (assessed using a 78D lens at the slit lamp and compared with standardised photographs based on the guidelines laid out in ${ }^{8}$ ), were fluent in the English language, had a mini-mental state examination (MMSE) score $>12$ and had capacity to consent to participation. Informed consent was obtained from all participants.

\section{Selection of a patient reported outcome measure}

Careful consideration was given to the selection of a suitable patient-reported outcome measure (PROM) for the current study. Guidance was taken from the Cochrane Health Related Quality of Life methods group ${ }^{9}$ and previous reviews of the use of PROMs in ophthalmology. ${ }^{10} 11$ The National Eye Institute 25 item Visual Functioning Questionnaire

${ }^{\mathrm{i}}$ Assumes $20 \%$ of patients from each of the age groups: $60-64,65-69,70-74,75-79$ and $80-84$ years with \% prevalence rates for dementia of $0.9,1.5,3.6,6.0$ and 12.2 , respectively. ${ }^{3}$ 
(VFQ-25) ${ }^{12}$ was selected for its favourable psychometric properties, its short administration time $(10 \mathrm{~min})$ and the breadth of concepts covered (including those about visual symptoms and functioning as well as the mental health and social influences of vision). The VFQ-25 has also been used widely in previously published literature and in a variety of different eye diseases (it is not specific to cataract). The VFQ-25 is scored from 0 (worst VQOL) to 100 (best VQOL).

\section{Assessment measures}

Participants were assessed preoperatively and at 1 year following first surgery. An interim appointment was also made at 3 months postoperatively if having single eye surgery or 2 months after second eye surgery if having sequential eye surgeries. Baseline assessment included demographic and medical questionnaire, clinical history from the participant and where available an informant detailing any cognitive symptoms, grading of lens opacities with the lens opacity classification system III (LOCSIII) ${ }^{13}$ and age-related macular disease (AMD) grading based on the guidelines of the international classification and grading system. ${ }^{8}$ At baseline, interim appointment and 1 year postoperatively the following assessments were performed: logarithm of minimum angle of resolution (logMAR) VA, Addenbrooke's Cognitive Examination (ACE-R; which includes the MMSE), ${ }^{14}$ National Eye Institute VFQ-25 ${ }^{12}$ and 15 item Geriatric Depression Scale (GDS-15). ${ }^{15}$ VA was defined as best $\log$ MAR VA in the better eye, corrected with up to date refraction and/or pinhole. The ACE-R is scored from 0 (worst cognition) to 100 (best cognition). The LOCSIII system for grading cataract scores four different aspects of lens opacity (nuclear colour, nuclear opalescence, cortical and posterior subcapsular) from 1 (no opacity) to 5 or 6 (most opacity). As a summary for cataract grade, we took the highest of these four scores for each eye and then used the grade from the eye with the least cataract (lower grade). AMD was graded as 0 (no changes), 1 (insignificant changes) or 2 (mild changes), and the grade from the least affected eye was used for analysis.

Participants were classified according to the predefined cut-offs on the ACE-R into normal cognition (ACE-R $\geq 88$ ) and impaired cognition (ACE-R <88). This cut-off has been reported to have a sensitivity of 0.94 and a specificity of 0.89 for detecting dementia. ${ }^{14}$ To aid clinical transferability of the results participants were also grouped according to whether they met clinical criteria for dementia or MCI. History taking and cognitive assessment from the participant and, where possible, an informant was taken by a clinician (JMJ). The diagnostic criteria for dementia from the Diagnostic and Statistical Manual V.4 were used to determine whether participants met the criteria for dementia ${ }^{16}$ and Petersen criteria were used to determine whether participants had MCI. ${ }^{17}$ Any dementia/MCI cases and borderline cases were discussed with an expert clinician (J-PT) to confirm or refute the diagnosis. To mimic clinical practice, no absolute cut-off values were used to define either dementia or MCI, but a whole clinical picture taking into account: the participant's level of functioning, ACE-R score, level of education and any report from participant, informant or another clinician that cognitive performance had changed from a previous level.

\section{Statistical analysis}

Statistical Package for Social Scientists (SPSS) V.17 was used for all the statistical analysis. p Values $<0.05$ were considered significant. Variables of interest were compared between cognitive groups and between those completing and not completing 1-year follow-up. Variables were compared using Pearson $\chi^{2}$ test (dichotomous variables), independent $t$ test (normally distributed variables) or Mann-Whitney U test (non-normally distributed variables).

Comparisons of visual measures (VQOL or VA) at baseline versus 1 year were made with paired t tests. Where significant differences were found, paired $t$ tests were used to compare 3-month postop measures to baseline and 1-year measures. Between-group comparisons were made with independent $t$ tests.

Analysis of covariance (ANCOVA) was used to compare outcome (VQOL or VA) at 1 year between cognitive groups while controlling for that measure at baseline. This approach was chosen as it generally gives greater statistical power than using the change from baseline or the percentage change as the outcome variable. The assumption of homogeneity of variance was checked using variance ratios compared against critical values for Hatley's $\mathrm{F}_{\max }{ }^{18}$

Linear regression models were used to assess the relationship between baseline cognition (predictor variable) and visual outcomes at 1 year (outcome variable) while correcting for potential co-predictors. The visual outcomes VQOL and VA were analysed separately and corresponding measures at baseline were entered as co-predictors. Other potential predictors were selected a priori if known to affect VA, VQOL and/or cognition as: age, gender, education, AMD grade, cataract grade, GDS-15 score and unilateral/sequential surgery. A backwards stepwise regression model was used to eliminate statistically redundant co-predictors. Multicollinearity and auto-correlation were checked for using tolerance statistics and Durbin-Watson test statistics, respectively. A normal probability plot of standardised residuals was used to ensure they were normally distributed. Individual cases with standardised residuals outside the range \pm 3.0 were identified and the regression model repeated excluding them.

The main analysis was based on a complete case analysis. To ensure losses to follow-up did not affect significantly the results, we also performed last outcome carried forward analyses where appropriate.

\section{RESULTS}

Figure 1 shows a summary of recruitment and follow-up numbers. A total of 112 participants were included in the baseline analysis, $99(88 \%)$ at the interim appointment and $91(81 \%)$ at 1 year. Of the 112 participants at baseline, 64 $(57 \%)$ had an ACE-R score $<88$ and were classified as having impaired cognition. For all participants, baseline mean ACE-R score (SD) was 83.7 (10.3). Of the three participants who died during the study follow-up (figure 1) all were in the impaired cognition group. There were significantly more participants completing follow-up in the normal cognition group (96\%) than in the impaired cognition group (70\%; Pearson $\chi^{2}$, $\mathrm{p}=0.001$ ). Table 1 shows the baseline characteristics for participants and a comparison between cognitive groups.

Of the 91 participants completing follow-up, 68 (74.7\%) had undergone bilateral sequential surgeries. There were no significant differences in the number of participants who had undergone bilateral surgeries between the impaired cognition and normal cognition groups (Pearson $\chi^{2}, \mathrm{p}=0.76$ ).

A total of nine $(8.0 \%)$ participants met a diagnosis of dementia and $23(20.5 \%)$ had MCI. Of these 32 participants with either dementia or MCI, only three (9\%) had previously been seen in a memory clinic.

Table 2 compares visual measures (VA and VQOL) between baseline and 1 year for the two cognitive groups. It also shows comparisons of VA and VQOL between cognitive groups. 
Figure 1 Recruitment and Retention flow- chart.

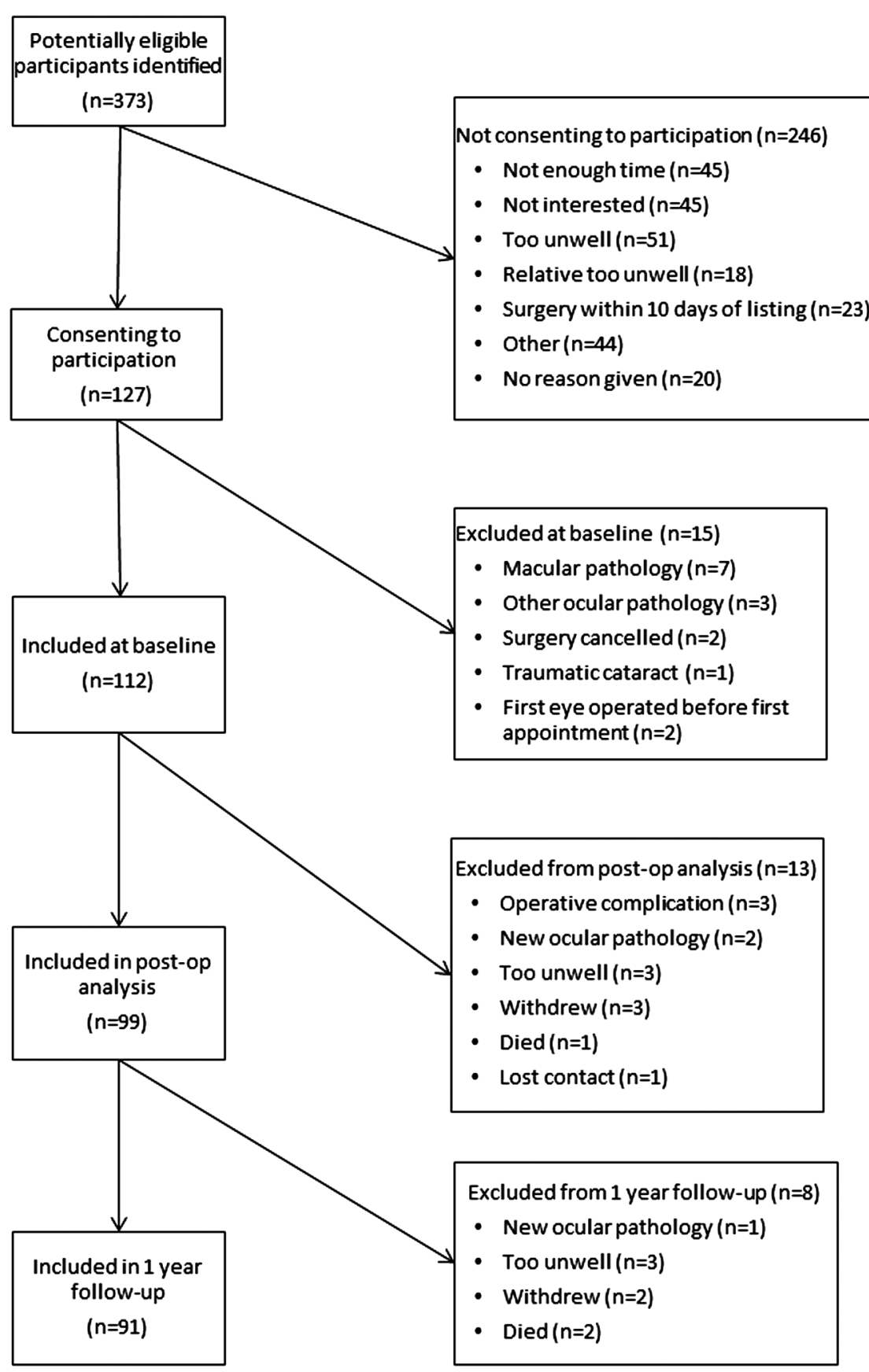

Significant differences between the two cognitive groups for VQOL $(\mathrm{F}=4.8, \mathrm{p}=0.03)$ and VA $(\mathrm{F}=4.9, \mathrm{p}=0.03)$ remained robust after the application of an ANCOVA with VQOL/VA at 1 year the dependent variable and VQOL/VA at baseline a covariate. Whole group analysis showed that participants $(n=91)$ had improved VQOL at 1 year compared with baseline (mean difference $13.5,95 \%$ CI 11.0 to 15.9 ) and the same was true for VA $(0.12,0.10$ to 0.15$)$. Interim analysis showed that VQOL improved at interim appointment compared with baseline (mean difference 11.9 , 95\% CI 9.6 to 14.2 ) and further improved at 1 year compared with interim (1.5, 0.0 to 3.1$)$. VA also improved at interim appointment compared with baseline (mean difference $0.12,95 \%$ CI 0.09 to 0.14 ) but not further at 1 year compared with interim $(0.00,-0.01$ to 0.02$)$.

When missing data at 1 year follow-up was imputed using a last outcome carried forward approach, paired t tests comparing 1-year follow-up with baseline for the 48 participants with normal cognition showed improved VQOL (mean difference 13.8, 95\% CI 10.5 to 17.0$)$ and improved VA $(0.12,0.09$ to 0.16$)$. Similarly, for the 64 participants with impaired cognition, comparisons between 1 year and baseline showed improvements in VQOL (mean difference $9.5,95 \%$ CI 6.4 to 12.6$)$ and VA $(0.11,0.07$ to 0.15$)$.

When participants were grouped according to the clinical diagnosis, those with dementia or MCI saw significant improvements in VQOL (mean difference 10.2, 95\% CI 2.8 to 17.5) and VA $(0.1,0.03$ to 0.16$)$. When compared with those not reaching a diagnosis of dementia or MCI, the dementia/MCI group had significantly poorer VQOL at 1 year (mean difference $5.2,95 \%$ CI 1.4 to 9.0 ) and significantly poorer VA at 1 year $(0.07,0.02$ to 0.12$)$.

Table 3 shows the results of the regression analyses. For each visual outcome, a summary of the initial model (including all 
Table 1 Demographics of participants at baseline comparing cognitive groups

\begin{tabular}{|c|c|c|c|}
\hline & \multicolumn{3}{|c|}{ Cognitive group } \\
\hline & $\begin{array}{l}\text { Normal } \\
(n=48)\end{array}$ & $\begin{array}{l}\text { Impaired } \\
(n=64)\end{array}$ & p Value* \\
\hline Age, mean (SD), years & $80.0(3.8)$ & $81.2(3.9)$ & 0.11 \\
\hline Gender, n (\%) men & $20(42)$ & $30(47)$ & 0.58 \\
\hline $\begin{array}{l}\text { Years full-time education, } \\
\text { median (IQR) }\end{array}$ & $11(10-13)$ & $10(9-11)$ & $<0.001$ \\
\hline $\begin{array}{l}\text { Depression score, GDS-15, } \\
\text { median (IQR) }\end{array}$ & $2(1-3.75)$ & $3(1-5)$ & 0.03 \\
\hline $\begin{array}{l}\text { Cognition score, ACE-R, } \\
\text { mean (SD) }\end{array}$ & $92.3(2.9)$ & $77.2(9.0)$ & $<0.001$ \\
\hline VFQ-25, mean (SD) & 79.9 (12.5) & $77.3(14.9)$ & 0.34 \\
\hline logMAR VA, mean (SD) & $0.13(0.09)$ & $0.21(0.17)$ & 0.002 \\
\hline AMD grade, median (IQR) & $0(0-1)$ & $0(0-0)$ & 0.45 \\
\hline Cataract grade, mean (SD) & $3.48(0.54)$ & $3.60(0.69)$ & 0.33 \\
\hline
\end{tabular}

${ }^{*} p$ Value refers to independent $t$ test, Pearson- $\chi^{2}$ or Mann-Whitney $U$ as appropriate. Note, that higher GDS-15 scores represent poorer mood, higher ACE-R scores represent higher cognition, higher VFQ-25 scores represent better functioning, higher logMAR scores represent worse VA, higher AMD grade indicates more macular changes and higher cataract grade represents more dense cataract.

ACE-R, Addenbrooke's Cognitive Examination; AMD, age-related macular degeneration; GDS-15, 15 item Geriatric Depression Score; logMAR, logarithm of minimum angle of resolution; VA, visual acuity; VFQ-25,25-item Visual Functioning Questionnaire.

predictors) and the final model (after backwards stepwise regression removed statistically redundant predictors) is shown. Cognition was a significant predictor of VQOL in the model but this became insignificant following the removal of outliers with absolute standardised residuals $>3$. Therefore, there remains uncertainty as to whether cognition was a significant predictor of VQOL. Cognition was, however, a significant predictor of VA, both in the initial and final regression models, with higher ACE-R score (better cognition) predictive of lower logMAR VA score (better vision; table 3 ).

\section{DISCUSSION}

In this group of older people attending for cataract surgery from the community, 64 (57\%) had ACE-R scores $<88$ and 32 (29\%) met the diagnostic criteria for dementia or MCI, suggesting that there are significant levels of cognitive impairment in a typical UK-based cataract clinic. Measures of VQOL and VA improved significantly for participants across all levels of cognition. However, poor cognition adversely affected VA outcomes and had a possible impact on VQOL outcomes.

We noted some significant differences in demographics between the two cognitive groups (see table 1), with lower levels of education in the impaired cognition group. This is not unexpected, as education and with it, poorer socioeconomic status is a known risk factor for cognitive impairment. ${ }^{19}$ Furthermore, those with lower educational level may struggle with certain aspects of cognitive assessment. We also saw lower mood in the cognitively impaired group. Poor mood may impair test performance on cognitive tasks due to poor concentration and motivation; depression is known to be associated with dementia and may be an independent risk factor for developing dementia. ${ }^{20}$ There was a difference in baseline VA between the two cognitive groups (table 1) but no differences in cataract density or macular changes. This supports the idea of a more direct relationship between vision and cognition, not simply as a result of higher levels of recognisable ocular comorbidity in those with lower cognition.

For participants in both groups, we saw a significant benefit following cataract surgery in terms of VQOL and VA. The mean improvements in VQOL of 12.4 (impaired cognition group) and 14.4 (normal cognition group) (see table 2) are clinically significant and similar to improvements reported previously following cataract surgery. ${ }^{21} 22$ The improvement in VA of 0.12 is equivalent to six letters (or just over one line) on the logMAR VA chart; this was significantly less than reported improvements in visual acuities seen in a recent $\mathrm{UK}$ audit, ${ }^{23}$ but this looked at improved vision for individual eyes (monocular vision) and not for patients (binocular vision) as we have done here. These results highlight the use of cataract surgery for patients in both normal and cognitively impaired populations.

Despite both groups seeing improvements in visual outcomes, there were significant differences between the groups in terms of VQOL and VA outcomes at 1 year (table 2). These differences remained when using an ANCOVA to correct for baseline VA/VQOL measures. Similar results were also seen when participants were grouped according to diagnosis (MCI or dementia) as opposed to cognitive score. While differences between impaired cognition and normal cognition groups in terms of VA and VQOL at 1 year were statistically significant, they were somewhat small and may not be clinically significant (table 2).

As well as considering participants divided into cognitive groups, we have examined cognition as a continuous variable in

Table 2 Visual outcomes at baseline and 1 year for the two cognitive groups

\begin{tabular}{|c|c|c|c|c|}
\hline & Baseline mean (SD) & 1 year mean (SD) & $\begin{array}{l}\text { 95\% } \mathrm{Cl} \text { for difference between } \\
\text { baseline and } 1 \text { year* }\end{array}$ & p Value* \\
\hline \multicolumn{5}{|l|}{ Outcome: visual quality of life } \\
\hline Normal cognition $(n=46)$ & $79.3(12.4)$ & $93.7(5.5)$ & 11.2 to 17.7 & $<0.001$ \\
\hline Impaired cognition $(n=45)$ & $77.6(13.9)$ & $90.0(9.6)$ & 8.6 to 16.3 & $<0.001$ \\
\hline $95 \% \mathrm{Cl}$ for difference between cognitive groups $t$ & -3.8 to 7.8 & 0.4 to 7.0 & & \\
\hline $\mathrm{p}$ Valuet & 0.5 & 0.03 & & \\
\hline \multicolumn{5}{|l|}{ Outcome: best corrected visual acuity } \\
\hline Normal cognition $(n=46)$ & $0.13(0.09)$ & $0.00(0.09)$ & 0.09 to 0.16 & $<0.001$ \\
\hline Impaired cognition $(n=45)$ & $0.18(0.14)$ & $0.06(0.11)$ & 0.08 to 0.16 & $<0.001$ \\
\hline $95 \% \mathrm{Cl}$ for difference between cognitive groups $t$ & 0.00 to 0.10 & 0.02 to 0.10 & & \\
\hline p Valuet & 0.05 & 0.007 & & \\
\hline
\end{tabular}


Table 3 Backwards stepwise regressions examining relationship between baseline cognition and visual outcomes while correcting for potential confounders

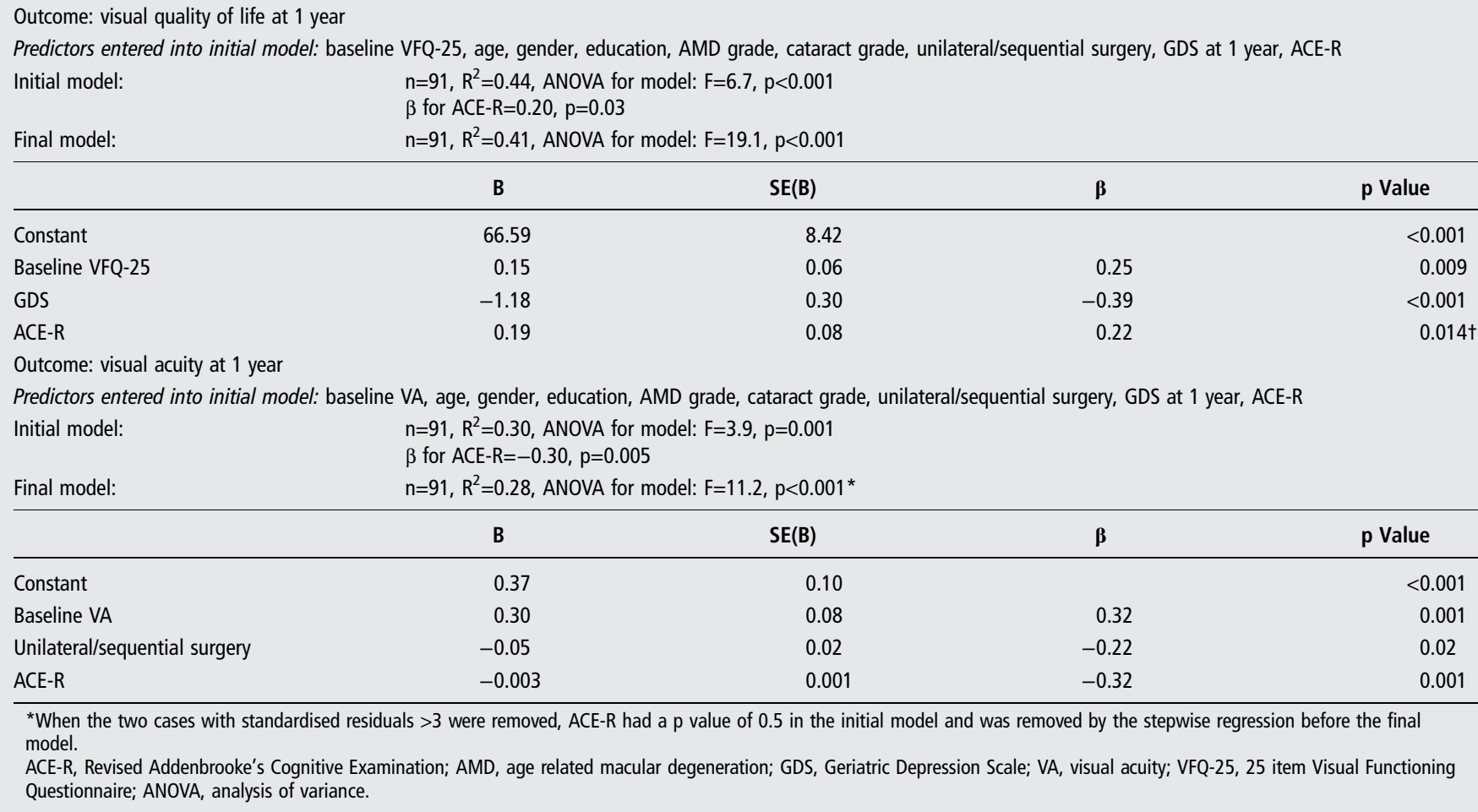

the regression analysis. While the dichotomisation of ACE-R scores is useful for clinical and statistical interpretation, studying cognition as a continuous variable affords greater statistical power. ${ }^{24}$ Regression analysis allows correction for potential confounders (eg, education/comorbid depression) and these analyses (table 3) confirmed a significant association between cognition and VA. The relationship between cognition and VQOL was less significant and not robust to removal of outliers. The association between cognition and vision cannot easily be explained by macular changes or denser cataract in those with cognitive impairment as there were no differences between cognitive groups in cataract or AMD measures (table 1), and they were corrected for in the regression model (table 3). Other possible explanations include higher levels of unrecognised pathology in those with lower cognition (eg, retinal or optic nerve pathology not identifiable by slit lamp biomicroscopy) ${ }^{25}{ }^{26}$; a common underlying degenerative aetiology for vision and cognitive impairment ${ }^{7}$ or an impact of cognition on attention, decision making and judgement skills required to read down a letter chart. $^{27}$ These factors could also account for the differences in VA seen between cognitive groups at baseline (see table 1).

In this sample of older people, $57 \%$ of participants fell below the ACE-R cut-off of 88 points, a significantly higher percentage than those meeting a diagnosis of dementia or MCI $(29 \%)$. It has been suggested that an ACE-R cut-off of $<88$ may be too stringent for day-to-day clinical practice and hence overestimate dementia, ${ }^{28}$ and this study would support this assertion. Our cohort was older than those used to describe these cognitive cut-offs and there is a lack of normative data on the ACE-R for people aged over 75 years. ${ }^{14}$ Our data does, however, suggest that there are significant levels of undiagnosed dementia within the eye clinic and thus ophthalmologists may have a role in initiating onward referrals.
Our recruitment and attrition rates (figure 1) are in line with other studies in similar populations. ${ }^{29} 30$ However, we saw significantly higher levels of attrition in the impaired cognition group and this may limit the generalisability of the results. To address the possibility that losses to follow-up were not completely at random (people with poor cognition or poor vision may find it harder to complete the study follow-up), we used a last outcome carried forward analysis which did not alter our conclusions. However, in this study, we have not considered those with severe cognitive impairment and thus the conclusions cannot necessarily be extrapolated to this patient group.

This research emphasises the use of cataract surgery in those with both normal and impaired cognition, with both groups experiencing significant improvements in visual outcomes. Cognitive impairment may, however, limit visual improvements following cataract surgery. Ophthalmologists helping patients to make decisions about whether to undergo cataract surgery need to take into account cognitive impairment, in the same way that older age and ocular comorbidities are considered as factors that may limit visual outcomes from cataract surgery. Meanwhile, further research is needed to understand the relationship between vision and cognition we have noted. It could be due to poor attention and concentration during VA testing in those with reduced cognition, as opposed to truly poorer vision suggesting that different approaches to accurately assess VA in patients with suspected cognitive impairment are needed.

Acknowledgements We thank Karen Morgan and Jackie Greenwood for their help in recruitment, Bianca Tobin for her help in participant assessments, Matthew Linsley for his assistance with statistical analysis and Elizabeth Hill for data checking. We thank Daniel Collerton and Urs Mosimann for their expertise and advice at the study design stage. We would also like to thank the Dementias and Neurodegenerative 
Diseases Research Network (DeNDRoN) for supporting the study and the staff at the Clinical Ageing Research Unit (CARU).

Contributors JMJ, MPC and J-PT designed the study question, interpreted the results and wrote the paper. JMJ collected and analysed the data. All authors had full access to all of the data. All authors contributed to and approved the final manuscript. Karen Morgan and Jackie Greenwood assisted with recruitment of participants. Bianca Tobin helped with participant assessments. Matthew Linsley provided statistical support. Elizabeth Hill checked data entry was accurate. Daniel Collerton and Urs Mosimann provided advice and expertise at the study design stage.

Competing interests This work was funded by a National Institute for Health Research (NIHR) Doctoral Research Fellowship award to JMJ. J-PT was supported by a Wellcome Trust Intermediate Fellowship. The research was supported by the NIHR Newcastle Biomedical Research Centre based at Newcastle upon Tyne Hospitals NHS Foundation Trust and Newcastle University. The NIHR had no role in the design or conduct of this research. The views expressed in this publication are those of the authors and not necessarily those of the NHS, the NIHR or the Department of Health.

Patient consent Obtained.

Ethics approval North East County Durham and Tees Valley Research Ethics Committee.

Provenance and peer review Not commissioned; externally peer reviewed.

Open Access This is an Open Access article distributed in accordance with the terms of the Creative Commons Attribution (CC BY 4.0) license, which permits others to distribute, remix, adapt and build upon this work, for commercial use, provided the original work is properly cited. See: http://creativecommons.org/ licenses/by/4.0/

\section{REFERENCES}

1 Cullen KA, Hall MJ, Golonsinskiy A. Ambulatory surgery in the United States, 2006. US Department of Health and Human Services Centres for Disease Control and Prevention, National Centre for Health Statistics, 2009.

2 The Information Centre for Health and Social Care. Hospital Episode Statistics, Admitted Patient Care, England-2012-13. 2013 [cited 201414 February 2014]. http://www.hscic.gov.uk/

3 Ferri CP, Prince M, Brayne C, et al. Global prevalence of dementia: a Delphi consensus study. Lancet 2005;366:2112-17.

4 Ward A, Arrighi HM, Michels $S$, et al. Mild cognitive impairment: disparity of incidence and prevalence estimates. Alzheimers Dement 2012;8:14-21.

5 Westcott MC, Tuft SJ, Minassian DC. Effect of age on visual outcome following cataract extraction. Br J Ophthalmol 2000;84:1380-82.

6 Wong TY. Regular review: effect of increasing age on cataract surgery outcomes in very elderly patients. BMJ 2001;322:1104-06.

7 Jefferis JM, Mosimann UP, Clarke MP. Cataract and cognitive impairment: a review of the literature. Br J Ophthalmol 2011;95:17-23.

8 Bird AC, Bressler NM, Bressler SB, et al. An international classification and grading system for age-related maculopathy and age-related macular degeneration. The International ARM Epidemiological Study Group. Surv Ophthalmol 1995;39:367-74.
9 Patrick D, Guyatt G, Acquadro C. Chapter 17: Patient-reported outcomes. In: Higgins JPT, Green S, eds. The Cochrane Collaboration. Cochrane Handbook for Systematic Reviews of Interventions Version 5.1.0, 2011.

10 de Boer MR, Moll AC, de Vet HC, et al. Psychometric properties of vision-related quality of life questionnaires: a systematic review. Ophthalmic Physiol Opt 2004;24:257-73.

11 McAlinden C, Gothwal VK, Khadka J, et al. A head-to-head comparison of 16 cataract surgery outcome questionnaires. Ophthalmology 2011;118:2374-81.

12 Mangione CM, Lee PP, Gutierrez PR, et al. Development of the 25-item National Eye Institute Visual Function Questionnaire. Arch Ophthalmol 2001;119: 1050-8.

13 Chylack LT Jr, Wolfe JK, Singer DM, et al. The lens opacities classification system III. The longitudinal study of cataract study group. Arch Ophthalmol 1993;111: 831-6.

14 Mioshi E, Dawson K, Mitchell J, et al. The Addenbrooke's Cognitive Examination Revised (ACE-R): a brief cognitive test battery for dementia screening. Int J Geriatr Psychiatry 2006;21:1078-85.

15 Yesavage JA. Geriatric depression scale. Psychopharmacol Bull 1988;24:709-11.

16 American Psychiatric Association. Diagnostic and statistical Manual for Mental Disorders, 4th edn, Text Revision (DSM-IV-TR). In: American psychiatric association. Arlington, VA: American Psychiatric Publishing, 2000:135-81.

17 Petersen RC. Mild cognitive impairment as a diagnostic entity. J Intern Med 2004;256:183-94.

18 Field A. Discovering statistics using SPSS. 3rd edn. London: SAGE Publications Ltd, 2009.

19 Shadlen M-F, Siscovick D, Fitzpatrick AL, et al. Education, cognitive test scores, and black-white differences in dementia risk. J Am Geriatr Soc 2006;54:898-905.

20 Jorm AF. Is depression a risk factor for dementia or cognitive decline? A review. Gerontology 2000;46:219-27.

21 Ishii K, Kabata T, Oshika T. The impact of cataract surgery on cognitive impairment and depressive mental status in elderly patients. Am J Ophthalmol 2008;146: 404-9.

22 Kaplan RM, Tally S, Hays RD, et al. Five preference-based indexes in cataract and heart failure patients were not equally responsive to change. J Clin Epidemiol 2011;64:497-506.

23 Sparrow JM, Taylor H, Qureshi K, et al. The Cataract National Dataset electronic multi-centre audit of 55,567 operations: risk indicators for monocular visual acuity outcomes. Eye 2012;26:821-6.

24 Altman DG, Royston P. The cost of dichotomising continuous variables. BMJ 2006;332:1080

25 Moreno-Ramos T, Benito-Leon J, Villarejo A, et al. Retinal nerve fiber layer thinning in dementia associated with Parkinson's disease, dementia with Lewy bodies, and Alzheimer's disease. J Alzheimers Dis 2013;34:659-64.

26 Sadun AA, Bassi CJ. Optic nerve damage in Alzheimer's disease. Ophthalmology 1990;97:9-17.

27 Das M, Bennett DM, Dutton GN. Visual attention as an important visual function: an outline of manifestations, diagnosis and management of impaired visual attention. Br J Ophthalmol 2007;91:1556-60.

28 Larner AJ. Addenbrooke's Cognitive Examination-Revised (ACE-R) in day-to-day clinical practice. Age Ageing 2007;36:685-6.

29 McMurdo ME, Roberts $\mathrm{H}$, Parker $\mathrm{S}$, et al. Improving recruitment of older people to research through good practice. Age Ageing 2011;40:659-65.

30 Davies K, Collerton JC, Jagger $C$, et al. Engaging the oldest old in research: lessons from the Newcastle 85+ study. BMC Geriatr 2010;10:64 